\title{
A micro-optical system for endoscopy based on mechanical compensation paradigm using miniature piezo-actuation
}

\author{
Pietro Cerveri*, Cynthia Corinna Zazzarini, Paolo Patete, Guido Baroni \\ Department of Electronics, Information and Bioengineering, Politecnico di Milano University, Milan 20133, Italy
}

Received 29 April 2013

Received in revised form 9 December 2013

Accepted 13 February 2014

\section{Introduction}

Recent advances in mini-invasive surgical techniques [1-3], as single port surgery (SPS) and natural orifice trans-luminal endoscopic surgery (NOTES), have been pushing the biomedical research toward the study of a next generation of feasible service tools for intra-corporal vision and tissue manipulation. These tools potentially can overcome the limitations, recognized in such new techniques, of conventional rigid laparoscopic instrumentation [47]. Basically, they are mainly passive instruments using optical fibers to deliver the light into the abdominal cavity and rod lenses to transmit the image back to the external camera sensor. Flexible endoscopes, ordinarily adopted in diagnostics, have been endeavored in substitution to rigid laparoscopes. They have a steerable tip to ease navigation, to direct the camera and the instruments. However, only the deflection of the tip can be controlled by means of steel cables, while the rest of the body is passive. Basically, flexible endoscopes are unsuitable for SPS and NOTES, being documented to suffer from both manipulation and vision issues [8]. For example, controlling the stiffness of the flexible endoscope is not trivial with effects on accurate force exertion. The number of tools that can be simultaneously used (number of tool channels) is one or two, and those tools are parallel and at close distance, impairing tool triangu-lation. Moving the axial position of the endoscope tip, to adapt the field of view of the surgical target, is not facilitated and still requires extra expert assistance. In order to overcome such drawbacks, sev-eral research developments of innovative surgical platforms, based on micro-robotic solutions, have been carried out [9-13]. As for the vision facility, there have been R\&D efforts in the direction of providing into a single distal device the main functional features such as scene illumination, imaging, target variable magnification (variable focal length) and vision orientation.

As far as the magnification is concerned, conventional zoom imaging systems are composed of several optical lens sets and some of them are variable in position. Mechanical compensation (MC) allows optical zoom with two mobile lens sets [14]. A coor-dinated motion of the two lens sets makes possible to change the magnification ("variator" lens set) while preserving the image focus ("compensator" lens set). However, moving to miniaturization, the

\footnotetext{
* Corresponding author. Tel.: +39 0223993352 
accurate control of the lens coordinated motion requires ad-hoc actuators. For example, considering a $10 \mathrm{~mm}$ optical system length, such an approach requires micro-metric lens positioning resolution $(\sim 10 \mu \mathrm{m})$, which is difficult to achieve with traditional electrome-chanical motors. In order to overcome the need of moving two lens sets, the combination of a single-moving-optical element and an extended-depth-of-focus method was proposed, which yielded a zoom factor of about $2.5 \times$ for an overall optical system length less than $12 \mathrm{~mm}$ [15]. Despite technically easier than traditional MC, the extended-depth-of-focus method required a cubic phase mask (spatial phase modulation function in the aperture stop of the lens system) and additional digital image restoration processes to recover the defocus effect [16].

In order to cope with miniaturization and accurate position control issues, shape memory alloys (SMA) and piezo actua-tion were proposed. SMA actuators were largely investigated as founding technology in miniaturized robotic systems for their significant displacement and force generation [17]. For exam-ple, in $[18,19]$, the authors presented an actuation system based on miniaturized SMA wire on silicon $(4.5 \mathrm{~mm} \times 1.8 \mathrm{~mm})$ able to generate a displacement of about $600 \mu \mathrm{m}$. Similarly in performance and size, piezo-motors have been recently successfully proposed for biomedical applications where the resolution and accuracy of the instrument positioning are demanding [20-23]. In [21], the authors proposed a piezoelectric ultrasonic microactuator $(1.5 \mathrm{~mm} \times 1.5 \mathrm{~mm} \times 5 \mathrm{~mm})$ able to generate a force of about $30 \mathrm{mN}$ for driving optics with potential applications in mobiles and micro-cameras. A linear piezo-motor $(2.8 \mathrm{~mm} \times 2.8 \mathrm{~mm} \times 6 \mathrm{~mm})$ (Squiggle ${ }^{\circledR}$ SQL-RV-1.8, Newscale Technologies Inc., Victory, NY, USA) was adopted to actuate a mini-robot in cardiac surgery [22] and a distractor in jaw bone surgery [23]. Interestingly, this commercial microactuator is able to exert a force of $300 \mathrm{mN}$ along a travel path of $6 \mathrm{~mm}$ with a nominal positioning resolution of $0.5 \mu$ $\mathrm{m}$, and overall overcomes $(3.3 \mathrm{~V})$ the high voltage power sup-ply requirement typical $(\sim 100 \mathrm{~V})$ of the traditional piezoelectric crystals.

In this paper, we describe the optical design of an innovative miniaturized camera, to be integrated within a robotic endoscope, which provides zoom capability thanks to mechanical compensation. A closed-loop actuation unit, devoted to properly and accurately move the lenses, was implemented and tested in a prototypical setup, by using Squiggle piezo-motors and magnetic position encoders (NSE-5310, AMS, Unterpremstaetten, Austria).

\section{Miniaturized optical system design}

The main requirements of endoscopic vision in mini-invasive abdominal surgery [8] were mainly taken into consideration for the optical design and then translated into a set of technical specifica-tions (Table 1). A distance range from the vision tool to the surgical target ranging from about 50 to $150 \mathrm{~mm}$ and a corresponding depth of field range in between 5 and $20 \mathrm{~mm}$ are common vision require-ments. Typically a laparoscope as an angular field of view (FOV) of about $70^{\circ}-80^{\circ}$. At an object distance of $50 \mathrm{~mm}$, an angular field of view (FOV) of about $50^{\circ}$, corresponding to a transversal object size of about $60 \mathrm{~mm}$, is usually considered adequate. Chromatic qual-ity of the resulting image is a significant feature for diagnostic and tissue discrimination purposes as well. As the aperture is essen-tial because it determines the resolution of the optical system, we setup its value by defining the stop surface diameter.

The main environmental constraint was represented by the size of the surgical incision, which typically ranges from 10 to $15 \mathrm{~mm}$. According to such requirements, a maximum external radius of the camera of $10 \mathrm{~mm}$, with maximum length of $30 \mathrm{~mm}$ was attempted. Assuming the lens support of at least $1 \mathrm{~mm}$ in thickness, a

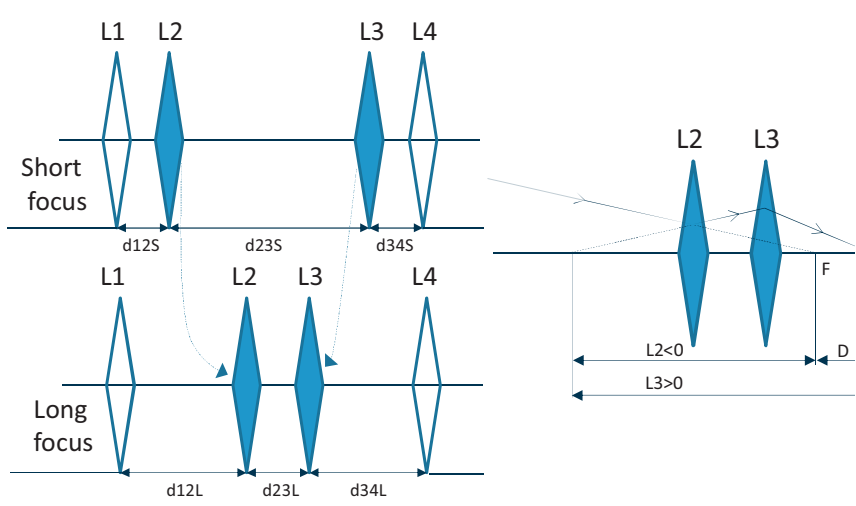

(a)

(b)

Fig. 1. Schematic diagram of a mechanical compensation system (a). L1 is the fron fixed set, L2 is the mobile zoom set, L3 is the mobile compensation set and L4 is the rear fixed set. The set distances at short focus and long focus are $d_{12 s}, d_{23 S}, d_{34 S}$, and $d_{12 L}, d_{23 L}, d_{34 L}$, respectively. Zoom and compensation sets (b). $F$ and $F^{\prime}$ designate object spot of the zoom set and image spot of the compensation set. During mechanical motion $D$ should be held constant.

maximum lens diameter of $7 \mathrm{~mm}$, a minimum lens edge of $0.5 \mathrm{~mm}$ and an overall optical system length lesser than of $20 \mathrm{~mm}$, were setup, as well. The design of the optical zoom took also into account the maximum travel path of the selected micro-motors $(6 \mathrm{~mm})$

In this study, the mechanical compensation (Fig. 1) was implemented using four lens sets, two fixed and two mobile, in the positive-negative-positive-positive optical format (see Appendix). The relations among the set distances $d_{12}, d_{23}, d_{34}$ were determined in function of the optical features required to the system.

The initial design was optimized, considering lens radius and inter-component separations, lens distances, even glasses, by using Optalix software (Optenso, $\mathrm{CH}$ ). A stop surface diameter of $1 \mathrm{~mm}$ was defined. Along with the various identified requirements (Table 1), astigmatism, transverse and longitudinal aberrations, and distortion quantities were opportunely included in the optimiza-tion for minimization. According to basic manufacturing tolerances, a lens edge less than $500 \mu \mathrm{m}$ was not allowed. No aspheric sur-faces were employed in the design. The best compromise between optical performances and surgical needs leaded to an optical sys-tem composed by 14 lenses, with a total length of $15.55 \mathrm{~mm}$, and an overall EFL ranging from about 1.5 to $4.3 \mathrm{~mm}$ (zoom factor: $2.8 \times$ ) (Fig. 2). Set I (distal lenses), featuring a wide aperture angle, consisted of the coupling between a negative doublet (glasses: SF4, SF5) and a convex lens (glass: LAF2), with a resulting positive focal length $(36.93 \mathrm{~mm})$. The negative doublet reduced the chromatic aberrations, whereas the convex lens, with high dioptric power, provided focus capability at lower distances. This allowed to get closer to the set II and reduce the overall length of the optical system.

The mobile zoom set II, consisting in a positive meniscus (glass: LAF2) and a negative doublet (glasses: LAF3, SF8) with a negative focal length $(-4.96 \mathrm{~mm})$, provided focal length adaptation of the whole optical system varying the aperture angle without affect-ing the image height. The mobile compensation set III, keeping the image focus without varying the magnification factor, consisted in a negative meniscus (glass: FK5) and a positive doublet (glasses: SF15, BASF64), with a positive focal length $(2.53 \mathrm{~mm})$. Set IV, devoted to the final image focus, consisted in a negative doublet (glasses: SF4, SF4), a positive meniscus (glass: LAF2), and a final pos-itive doublet (glasses: LAF2, SF4). This last set was characterized by a positive focal length $(17.17 \mathrm{~mm})$ and a high dioptric power. The predicted lens travel (Fig. 3) was almost linear for the compensation 
Table 1

Main constraints and specifications of the optical system.

\begin{tabular}{|c|c|c|c|}
\hline \multicolumn{2}{|l|}{ Operational constraints } & \multicolumn{2}{|c|}{ Optical and technical specifications } \\
\hline Maximum internal diameter & $8 \mathrm{~mm}$ & Optical system length & $<20 \mathrm{~mm}$ \\
\hline Maximum camera length & $30 \mathrm{~mm}$ & Lens diameter & $2 \mathrm{~mm} \leq \varnothing \leq 7 \mathrm{~mm}$ \\
\hline Working distance & $50-150 \mathrm{~mm}$ & Lens edge & $\geq 0.5 \mathrm{~mm}$ \\
\hline Depth of field & $5-20 \mathrm{~mm}$ & Stop surface $\varnothing$ & $\overline{1} \mathrm{~mm}$ \\
\hline Image sensor height & $1.53 \mathrm{~mm}$ & Optical distortion & $<15 \%$ \\
\hline Wavelengths & RGB & Field of view (FOV) & $50^{\circ}-60^{\circ}$ \\
\hline Maximum image distortion & $25 \%$ & Optical zoom & $\sim 3 \times$ \\
\hline Maximum motor travel & $6 \mathrm{~mm}$ & Lens displacement & $\leq 6 \mathrm{~mm}$ \\
\hline
\end{tabular}

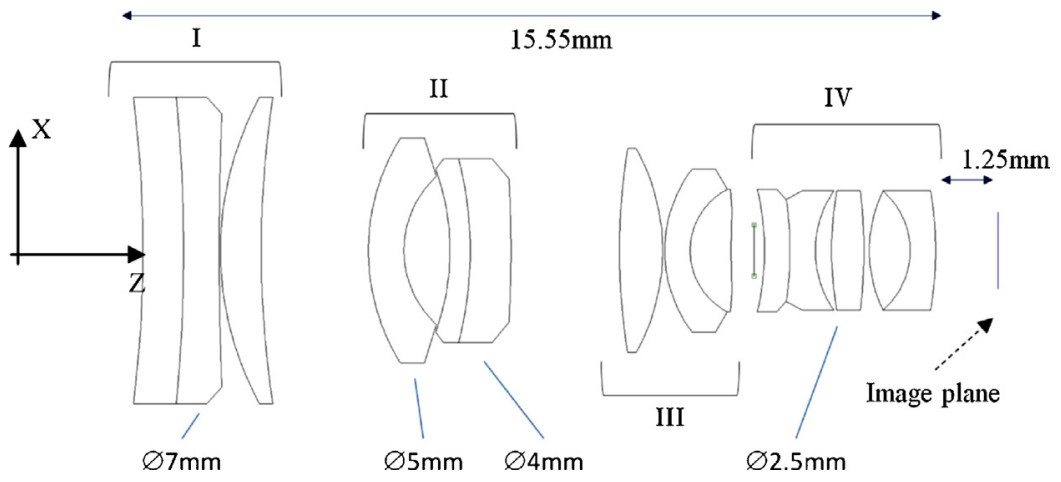

Fig. 2. Layout of the lens system. Lens sets II and III are mobile.

set (path: $0.98 \mathrm{~mm}$ ) whereas it was non-linear for the zoom set (path: $3.30 \mathrm{~mm}$ ).

\section{Optical performance}

\subsection{Lens system}

Three different effective focal lengths (EFL), corresponding to wide-angle, mid-range and tele-photo zoom configurations, were taken into account (Fig. 4). In the wide-angle configuration the EFL, the $\mathrm{F}$ number and the FOV were $1.56 \mathrm{~mm}, 2.79^{\circ}$ and $56^{\circ}$, respectively. In mid-range configuration, we got $2.60 \mathrm{~mm}, 2.79^{\circ}$ and $32^{\circ}$, respectively. In tele-photo configuration, we got $4.31 \mathrm{~mm}, 2.76^{\circ}$

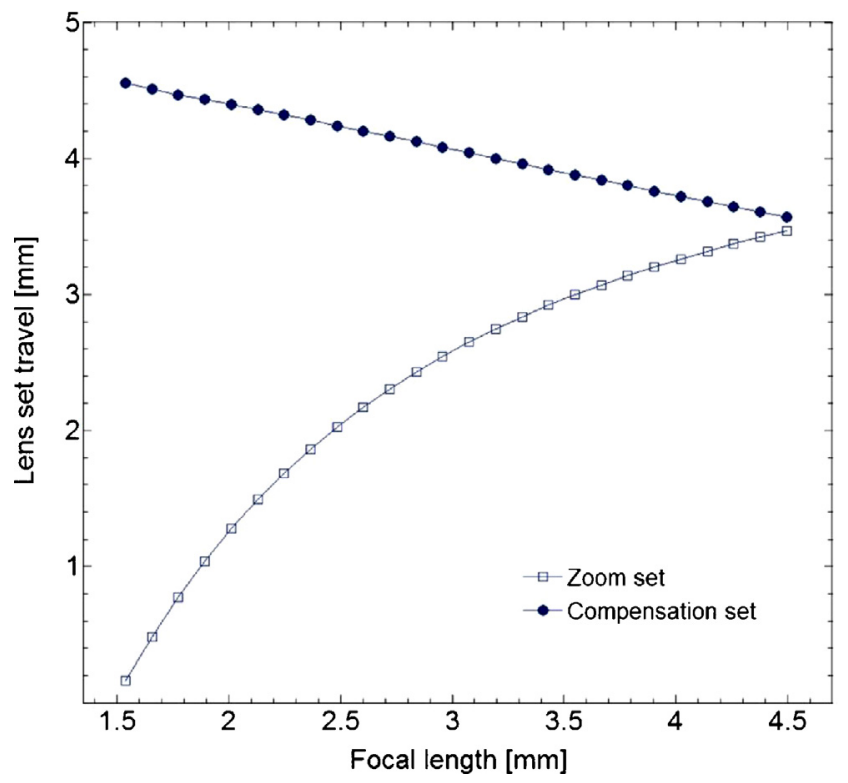

Fig. 3. The path chart of zoom set and compensation set.

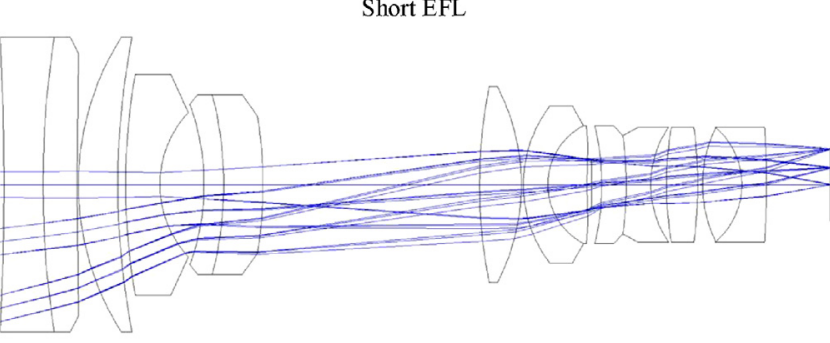

Mid EFL

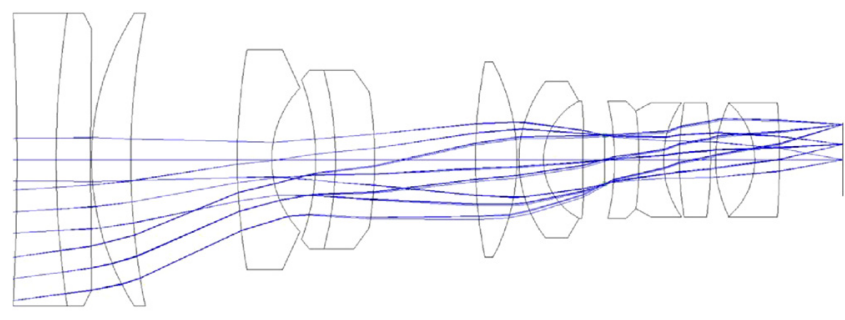

Long EFL

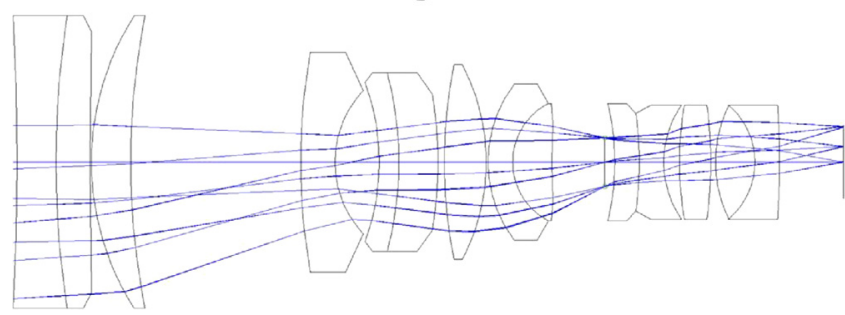

Fig. 4. Three reference zoom positions: wide-angle (short focal), mid-range (mid focal) and tele-photo (long focal). In wide-angle configuration, rays were casted at $0^{\circ}, 13^{\circ}$ and $28^{\circ}$. In mid-range configuration, rays were casted at $0^{\circ}, 7^{\circ}, 16^{\circ}$. In telephoto configuration, rays were casted at $0^{\circ}, 4^{\circ}$ and $9^{\circ}$. The object was assumed to be at $150 \mathrm{~mm}$ away from the distal lens set I. The image plane height was constant across the zoom range. 
Table 2

Longitudinal spherical aberration, optical distortion, and MTF.

\begin{tabular}{llll}
\hline & Wide-angle & Mid-range & Tele-photo \\
\hline $\begin{array}{c}\text { Max longitudinal } \\
\text { spherical aberration }\end{array}$ & $35 \mu \mathrm{m}\left(28^{\circ}\right)$ & $25 \mu \mathrm{m}\left(16^{\circ}\right)$ & $25 \mu \mathrm{m}\left(9^{\circ}\right)$ \\
$\begin{array}{c}\text { Max distortion } \\
\text { Perceived sharpness }\end{array}$ & $12 \%($ barrel $)$ & $6 \%($ barrel $)$ & $1 \%($ pincushion $)$ \\
$\quad$ (MTF at 50\%) & $84 \mathrm{lp} / \mathrm{mm}$ & $80 \mathrm{lp} / \mathrm{mm}$ & $65 \mathrm{lp} / \mathrm{mm}$ \\
& & &
\end{tabular}

and $18^{\circ}$, respectively. Under such conditions, the image height was almost constant $(\sim 1.50 \mathrm{~mm})$ across the zoom range (nominal height of the image sensor: $1.53 \mathrm{~mm}$ ).

The performance of the simulated optical system was evaluated in terms of astigmatism, transverse and longitudinal spherical aberrations, and optical distortion. In order to quantify the image contrast, the perceived image sharpness was adopted [24]. This measure is a practical parameter used to qualify the optical resolution and relates to the spatial frequency, represented by the modulation transfer function (MTF). ${ }^{1}$ Astigmatic field curves and transverse aberration, which was displayed through spots, were reported in Figs. 5-7, for the three zoom configurations and for three visible wavelengths. Considering achromatic astigmatism, the maximum difference between sagittal and tangential values was less than $15 \mu \mathrm{m}$ at the angular field of $28^{\circ}$ (half FOV) in wideangle configuration. Considering the three wavelengths separately, the maximum predicted chromatic astigmatism was less than $25 \mu \mathrm{m}$ in all the three zoom configurations.

As far as transverse aberration is concerned, the maximum value was less than $15 \mu \mathrm{m}$. This value was compared to the nominal Airy disk diameter defined as 14:

$\varnothing_{\text {Airy }}=2.44 \lambda F \#$

where $\lambda$ and $F \#$ are the light wavelength and the $F$ number of the optical system. Traditionally, an aberration less than 10 times the Airy disk diameter is considered acceptable [14]. Assuming an average $F \#$ of about 3 and the smallest wavelength $(\lambda=0.420 \mu \mathrm{m})$, we obtained a nominal Airy disk of $3 \mu \mathrm{m}$. The predicted $15 \mu \mathrm{m}$ for the transverse aberration was than just 5 time greater than Airy disk diameter thus the predicted transverse aberration can be considered acceptable.

Results about longitudinal aberration, optical distortion and MTF were synthesized in Table 2. The optical distortion was maximum at wide-angle configuration (barrel: $16 \%$ ) and was lower at both middle range (barrel: 6\%) and telephoto (pincushion: 1\%) configurations. The prediction of the perceived sharpness at field boundary was $84 \mathrm{lp} / \mathrm{mm}$ at the wide-angle, $80 \mathrm{lp} / \mathrm{mm}$ at the midangle, and $65 \mathrm{lp} / \mathrm{mm}$ at tele-photo configurations. While such values are lower than the maximum nominal spatial frequency resolved by the CMOS ( $\sim 180 \mathrm{lp} / \mathrm{mm})$, they are consistent with typical MTF values of digital photography and sufficient to get an acceptable image quality [24].

The main optical and structural features of a traditional rigid laparoscope (Fig. 8) were considered and compared to the designed optical system (Table 3). As it can be seen, the optical design performed in this study overcomes the performances of a rigid laparoscope especially for astigmatism, aberration and distortion.

\footnotetext{
1 The essential meaning of MTF is rather simple. Suppose you have a pattern consisting of a pure tone (a sine wave). At frequencies where the MTF of an imaging system or a component (film, lens, etc.) is $100 \%$, the pattern is un-attenuated, i.e., it retains full contrast. The perceived sharpness, expressed in line pairs per millimeter $(\mathrm{lp} / \mathrm{mm})$, is the value where contrast has dropped by half, namely when the MTF reaches $50 \%$.
}

Table 3

Feature comparison between a typical laparoscope and the designed optical system.

\begin{tabular}{lll}
\hline Optical features & Laparoscope & Our system \\
\hline Total length & $>20 \mathrm{~cm}$ & $<2 \mathrm{~cm}$ \\
EFL & $2 \mathrm{~mm}$ & $1.56-4.31 \mathrm{~mm}$ \\
Magnification & & $2.8 \times$ \\
Max astigmatism & $125 \mu \mathrm{m}$ & $25 \mu \mathrm{m}$ \\
Max aberration & $100 \mu \mathrm{m}$ & $35 \mu \mathrm{m}$ \\
Distortion & $18 \%$ & $12 \%$ \\
Minimal MTF & $65 \mathrm{lp} / \mathrm{mm}$ & $65 \mathrm{lp} / \mathrm{mm}$ \\
\hline
\end{tabular}

\subsection{Uncertainty of the lens set positioning}

In order to evaluated the effect of the uncertainty of the lens set positioning on the optical aberrations, we systematically simulated progressive shifts (step: $10 \mu \mathrm{m}$ ) of the compensation and zoom sets independently in two separated tests, up to a positioning error of $\pm 70 \mu \mathrm{m}$.

Without loss of generality mid-range position was chosen and the achromatic astigmatism and transverse spherical aberration were predicted. In Fig. 9, the deviation results of the two optical aberrations were depicted. The reference values of the two nominal optical aberrations for the selected mid-range position were set to zero so that the deviation plots were referred to the corresponding values. The deviation trends were almost linear with the shifting. As expected, errors in positioning the compensation lens set were more significant in decreasing the image quality than an identical uncertainty in the positioning of the zoom lens set. Interestingly, just a deviation of $20 \mu \mathrm{m}$ of positioning error of the compensation lens set decreased the astigmatism quality of more than $40 \mu \mathrm{m}$. We extrapolated that a positioning error of $5 \mu \mathrm{m}$ would deteriorate the aberration with a maximum value of $10 \mu \mathrm{m}$.

\subsection{Effects of tolerances}

In order to evaluate the effect of the manufacturing tolerances, approximation of the lens axial alignments and opto-mechanics mounting, we simulated optical and mounting uncertainty. Without loss of generality, we considered separately the uncertainty of the lens set number II (zoom set), the lens set number III (compensation set) and the lens set number IV, disregarding the lens set I. Five different testing conditions were considered (Table 4) and the results compared to nominal optical performances.

A lens curvature variation of $\pm 1 \%$, with respect to the nomi-nal value, was given to simulate manufacturing tolerance (test T1). Given that we are dealing with absolute lens radii ranging from 1.5 to $15 \mathrm{~mm}$, the chosen tolerance corresponded to a metric range of about 15-150 $\mu \mathrm{m}$. The opto-mechanics mounting imprecision was simulated by modifying: (a) the axial distance between the posi-tive meniscus and the negative doublet for zoom set, between the negative meniscus and the positive doublet for compensation set, between the first two lenses (negative doublet and positive meniscus) and the final positive doublet for the lens set IV (test T2); (b) the axial alignment on the optical axis through a tilt angle about the transverse X axis (test T3); (c) the translation away from the optical

Table 4

Protocol for testing the effect of manufacturing tolerances. Test T1 simulated the manufacturing tolerance. Test T2 simulated opto-mechanics mounting imprecision. Test T3 simulated axial deviation of the optical axis. Test T4 simulated lens decentering.

\begin{tabular}{llllll}
\hline Lens tolerance & $\mathrm{T} 1$ & $\mathrm{~T} 2$ & $\mathrm{~T} 3$ & $\mathrm{~T} 4$ & $\mathrm{~T} 5$ \\
\hline Radius of curvature & $\pm 1 \%$ & 0 & 0 & 0 & $\pm 1 \%$ \\
Relative axial distance & 0 & $\pm 50 \mu \mathrm{m}$ & 0 & 0 & $\pm 50 \mu \mathrm{m}$ \\
X - Tilt & 0 & 0 & $\pm 1^{\circ}$ & 0 & $\pm 1^{\circ}$ \\
X - Decentering & 0 & 0 & 0 & $\pm 50 \mu \mathrm{m}$ & $\pm 50 \mu \mathrm{m}$ \\
\hline
\end{tabular}




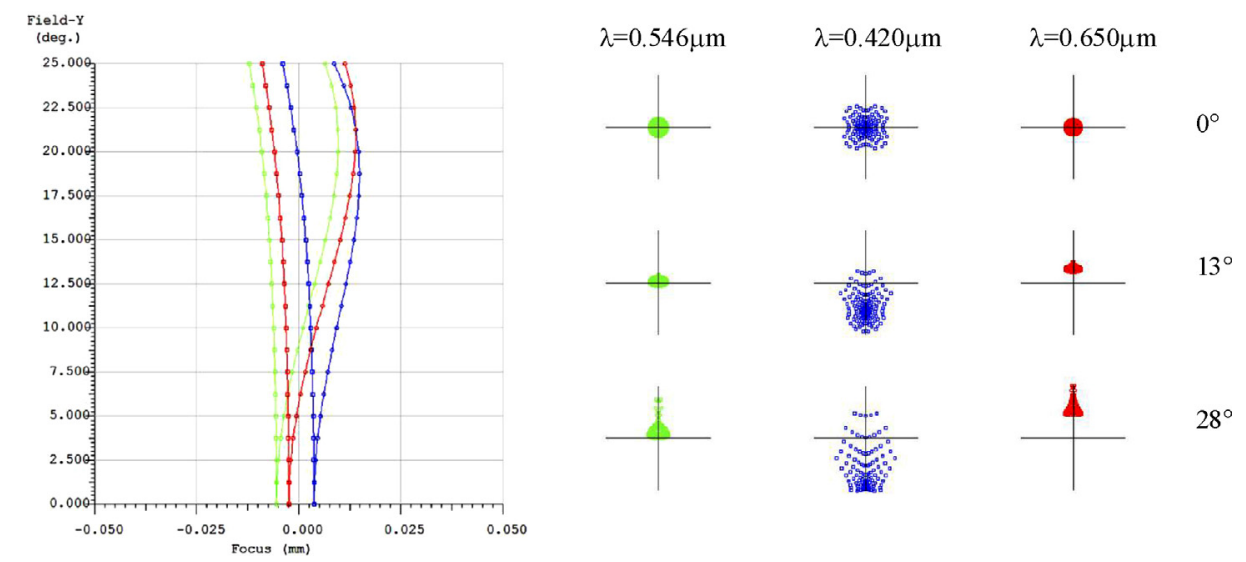

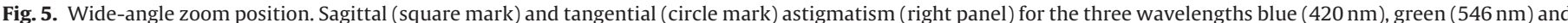

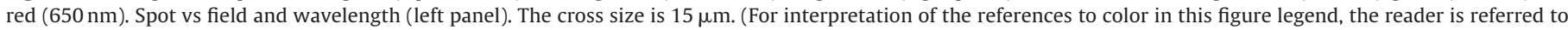
the web version of this article.)
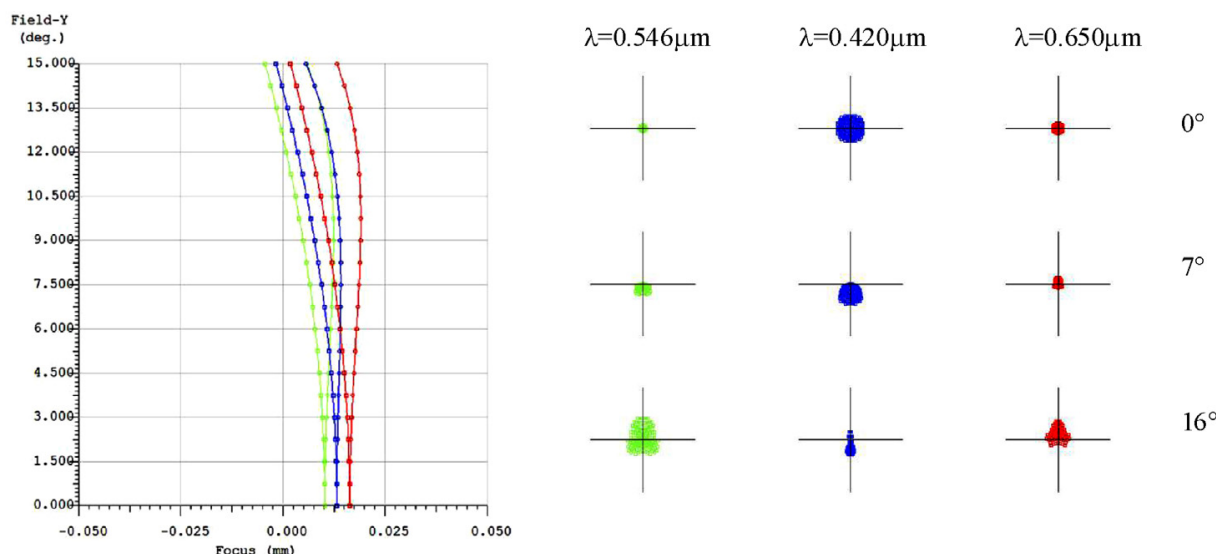

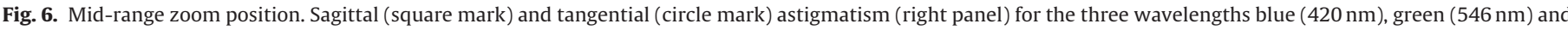

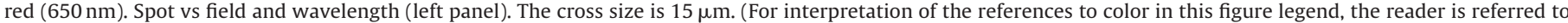
the web version of this article.)
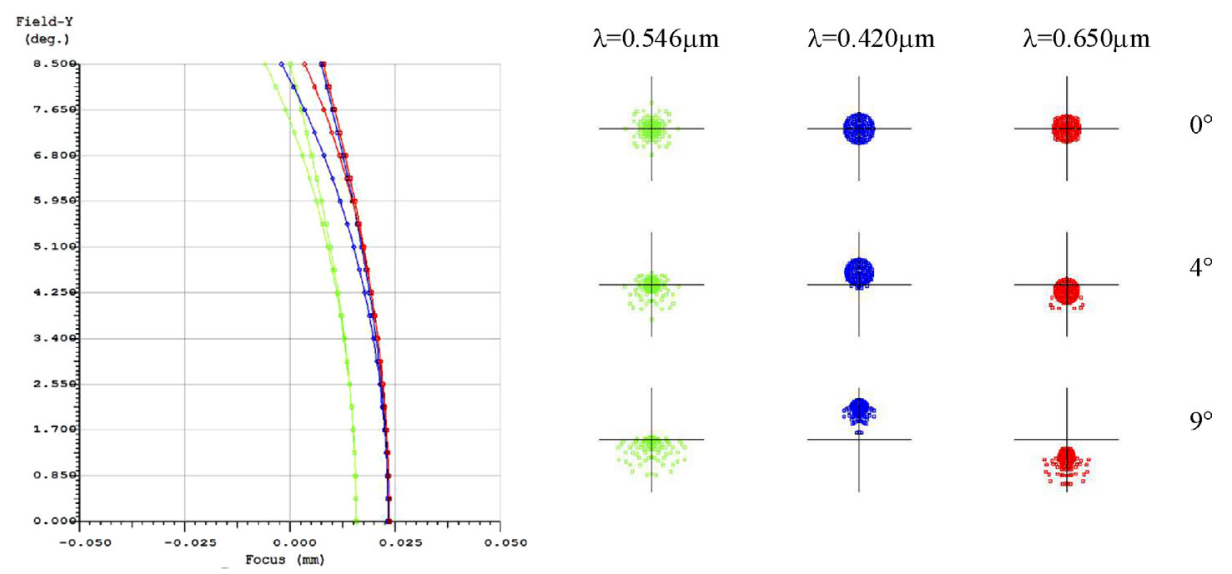

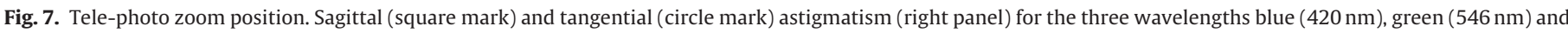

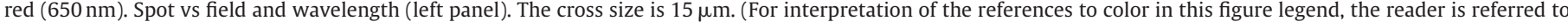
the web version of this article.)

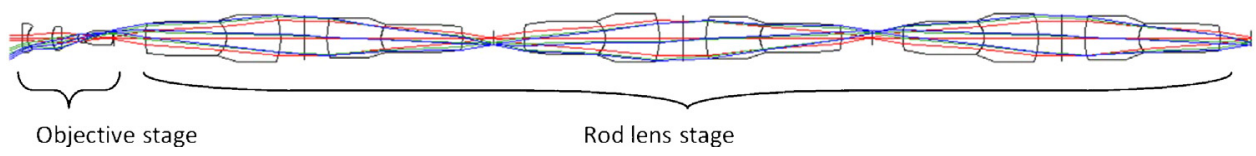

Fig. 8. Optical design of a rigid laparoscope. 

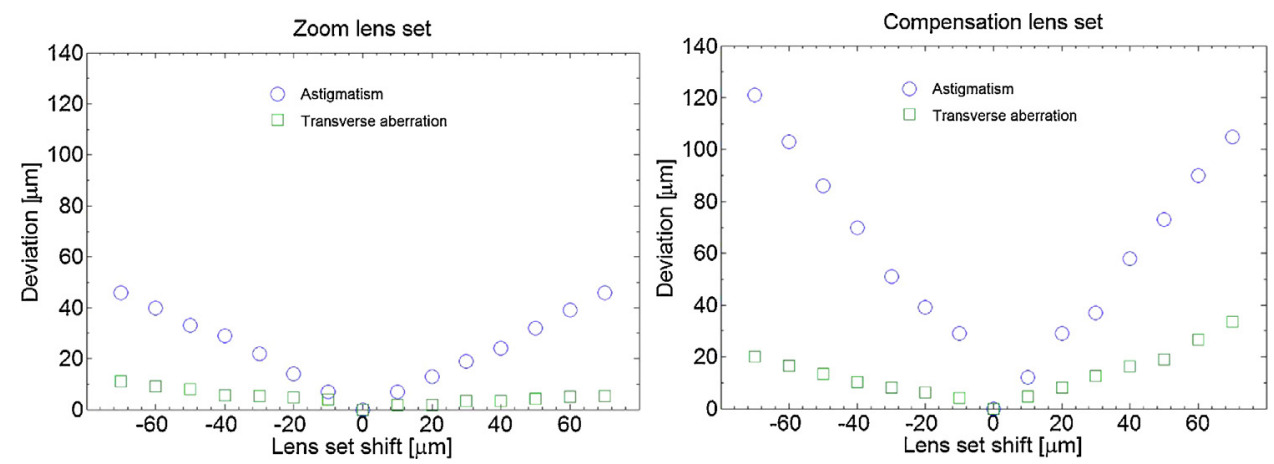

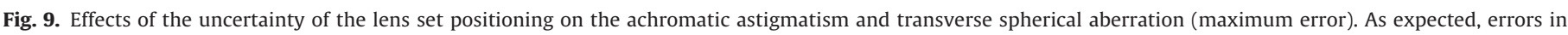
positioning the compensation lens set were more significant in decreasing the image quality.

Table 5

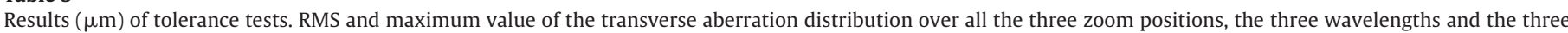
FOV. The nominal design values are 3.9 and $7.6 \mu \mathrm{m}$, respectively.

\begin{tabular}{|c|c|c|c|c|c|c|c|c|c|c|}
\hline \multirow[t]{2}{*}{ Lens set } & \multicolumn{2}{|l|}{$\mathrm{T} 1$} & \multicolumn{2}{|l|}{$\mathrm{T} 2$} & \multicolumn{2}{|l|}{ T3 } & \multicolumn{2}{|l|}{$\mathrm{T} 4$} & \multicolumn{2}{|l|}{$\mathrm{T} 5$} \\
\hline & - & + & - & + & - & + & - & + & - & + \\
\hline II (Zoom) & $4.4(11.4)$ & $4.3(10.1)$ & $4.5(13.3)$ & $4.3(12.1)$ & $4.7(12.3)$ & $4.3(11.9)$ & $4.5(12.6)$ & $4.4(11.9)$ & $5.6(16.2)$ & $5.4(15.9)$ \\
\hline III (Compensation) & $8.6(20.2)$ & $9.4(25.3)$ & $4.7(13.7)$ & $4.4(12.3)$ & $7.4(20.7)$ & $6.8(18.6)$ & $5.3(16.7)$ & $4.2(11.5)$ & $14.2(32.6)$ & $14.9(37.1)$ \\
\hline IV (Proximal) & $4.5(9.6)$ & $4.1(8.7)$ & $4.1(8.8)$ & $4.3(9.1)$ & $4.7(12.0)$ & $5.3(15.9)$ & $6.2(15.6)$ & $5.8(14.8)$ & $8.9(21.7)$ & $7.4(19.4)$ \\
\hline
\end{tabular}

axis through a decentering displacement in X direction (test T4). In test T5, all the previous effects were considered altogether.

Root mean square (RMS) and maximum value of the transverse aberration distribution over all the three zoom positions, the three wavelengths and the three FOV were used to test the tolerance effect. The nominal design values were 3.9 and $7.6 \mu \mathrm{m}$, respectively. Results showed that deviations from design are more critical in the compensation lens set than zoom lens set (Table 5). This was in agreement with previous results (see Section 3.1). In the lens set IV, the tolerance was more important than that of the zoom set but less critical than that of compensation lens set.

In the zoom set, the lens curvature tolerance (T1) increased the RMS of the transverse aberration of about $13 \%$ with respect to the nominal value. Tolerances of the other three quantities affected similarly the transverse aberration. Considering the joined effect of all the tolerances (T5), the RMS increased overall of about $40 \%$. In the compensation set, the lens curvature tolerance increased the RMS of about $130 \%$. The relative axial distance (T2) decentering displacement (T4) tolerances were similar, however less critical with respect to $\mathrm{T} 1, \mathrm{~T} 3$, and $\mathrm{T} 5$ tolerances. Considering the joined effect of all the tolerances (T5), the RMS increased overall of about $270 \%$. A relative increase of the RMS of about $150 \%$ was obtained between zoom and compensation sets (T5). In the lens set IV, the effect of the lens curvature tolerance was quite similar to that one in the zoom set. In contrast to the results of the compensation set, the tolerance of lens decentering (T4) affected more the transverse aberration than the lens tilt (T3).

A specific analysis was performed for the tolerance of the radius of curvature in the compensation set. Four tolerances were considered: $1 \%, 0.5 \%, 0.1 \%, 0.05 \%$ (Table 6 ). As it can be seen, the effect of

\section{Table 6}

Results $(\mu \mathrm{m})$ of tolerance of the radius of curvature in the compensation set. RMS and maximum value of the transverse aberration distribution over all the three zoom positions, the three wavelengths and the three FOV. The nominal design values are 3.9 and $7.6 \mu \mathrm{m}$, respectively.

\begin{tabular}{lclll}
\hline Lens set & $1 \%$ & $0.5 \%$ & $0.1 \%$ & $0.05 \%$ \\
III (Compensation) & $8.6(20.2)$ & $5.5(14.8)$ & $4.1(7.9)$ & $4.0(7.8)$ \\
\hline
\end{tabular}

the tolerance was critical in reducing optical performances when moving from $0.1 \%$ to $0.5 \%$.

\section{Actuation and position control performance}

For the actuation of the lens sets II and III, the piezo-electric Squiggle motor and magnetic position encoder, mentioned earlier in the text, were adopted. The Squiggle motor features variable speed from $1 \mu \mathrm{m} / \mathrm{s}$ up to about $10 \mathrm{~mm} / \mathrm{s}$, according to the duty cycle, with positioning resolution less than $1 \mu \mathrm{m}$ in an overall travel path of $6 \mathrm{~mm}$. The motor is based on a screw, which is moved back and forth within a threaded nut encapsulated among 4 piezo-electric plates. These are electrically driven by using 4 signals opportunely dephased and inverted. When the motor is holding the screw position there is no power consumption thanks to the motor auto switch-off feature. However, the piezo-motor is just able to push so that the retrograde motion must be facilitated using for example passive springs. The motor control can be performed by setting a register value (11 useful bits) corresponding to a pulse number $C$ that in turn codes for the linear motor speed. For example, value of 2047 with a duty cycle of $50 \%$ leads to a speed of $7 \mathrm{~mm} / \mathrm{s}$. The actuation is iteratively performed by defining the pulse counter and waiting up until the register is null, which corresponds to an executed motion step of length $D$. The magnetic position encoder, based on Hall effect, is able to measure linear movements with a resolution of $0.5 \mu \mathrm{m}$ that is in the same order of magnitude of the Squiggle motor motion resolution. Along with the magnetic sensor, the position encoder requires a passive part (magnetic strip) attached to the mobile support and positioned at a maximum distance of $300 \mu \mathrm{m}$ from the sensor.

In order to test the performance of the positioning, we arranged a prototypical experimental setup developing a printed control board $(\mathrm{PCB})$, equipped with one squiggle motor, the corresponding power driver, the position sensor and the corresponding magnetic strip (Fig. 10). All the active devices were connected to an on-board programmable micro-controller. The motor shaft was mechanically connected to a plastic manufacture emulating the mechanical support of the lens set. 


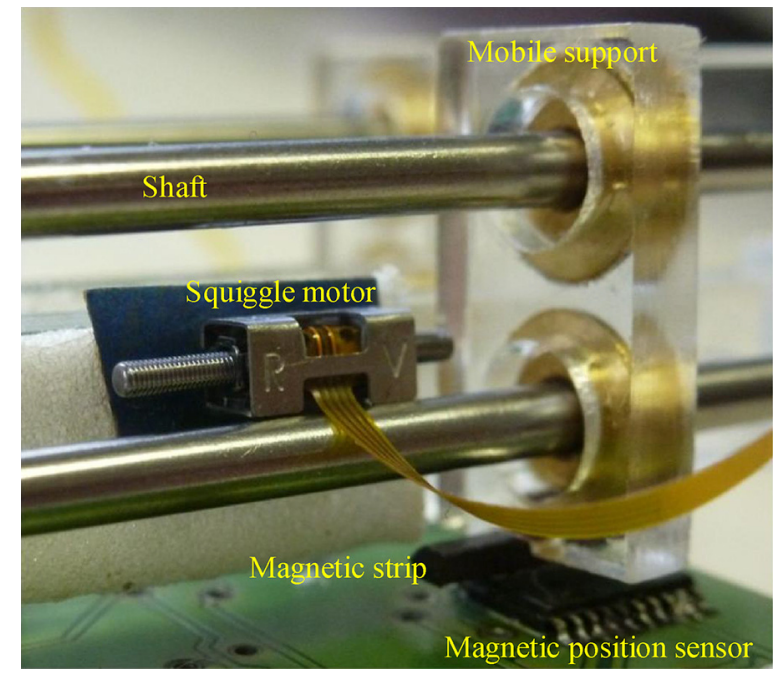

Fig. 10. Squiggle piezo-electric motor along with the position encoder and the magnetic strip in the testing setup. The distance between the magnetic strip and the sensor was in the range of about $300 \mu \mathrm{m}$.

The protocol for the calibration of the piezo-actuator involved 15 repetitions of a progressive motion of the motor screw at maximum speed from the rest position up to about $3 \mathrm{~mm}$ of travel (Fig. 11). The position of the mobile support (see Fig. 10) was mea-sured by the Hall sensor at a fixed time step of $35 \mathrm{~ms}$. As it can be seen, the position uncertainty grows as the travel path increase from a value of the standard deviation of about $64 \mu \mathrm{m}$ (at about $0.5 \mathrm{~mm}$ path) to a value of $292 \mu \mathrm{m}$ (at about $3 \mathrm{~mm}$ path). This can be motivated by considering the friction between the mobile sup-port and the shaft (see Fig. 10). While an almost linear relationship was found $\left(R^{2}=0.99, p\right.$-value $\left.<10^{-6}\right)$, the positioning accuracy was not in agreement with the requirements. Therefore, a proportional control loop was implemented to both increase the positioning accuracy and cope with the effects of the potential variable load on the motor shaft and the friction on the mobile components, which can invalidate the calibration relationship. In detail, the control loop was expressed as:

$c_{i}= \begin{cases}C & \text { if }\left|P_{T}-P_{i}\right|>D \\ k C \frac{\left|P_{T}-P_{i}\right|}{D} & \text { otherwise }\end{cases}$

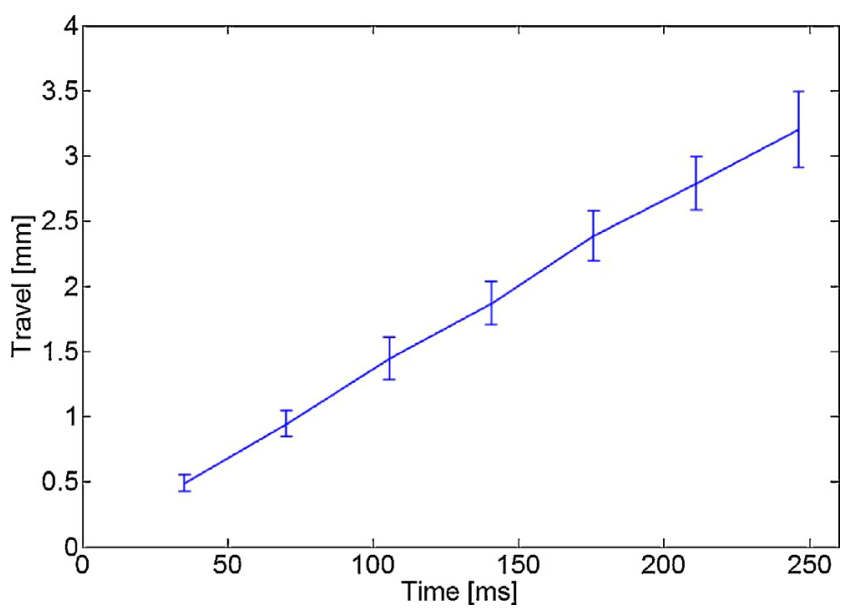

Fig. 11. Calibration of the piezo-actuator. The position of the motor was measured by the Hall sensor at fixed time steps $(35 \mathrm{~ms})$. Corresponding error bars, computed over 15 repetitions, were reported. where $c_{i}, k, P_{T}, P_{i}, D$ are the current pulse number to drive the motor at iteration $i$, the proportional factor less than 1 , the desired travel, the measured travel, nominal travel $D(\sim 150 \mu \mathrm{m})$ for the maximum pulse number, estimated by calibration, respectively.

Without lack of generality, the control loop was implemented to stop the motion when the magnetic position encoder measured a difference between the measured and the desired travel less than a threshold $s(5 \mu \mathrm{m})$. According to simulation results of the positioning uncertainty (Fig. 9), this choice was done to tolerate a maximum decrease of the aberrations less than $10 \mu \mathrm{m}$. Five different length of the traveling path, namely $0.25,0.5,1,2$ and $4 \mathrm{~mm}$, were tested. Control results using $k=0.5$ (Fig. 12) showed that all the travel paths reached the desired positions and were approximately linear in time except when approaching the desired position (red circles in Fig. 12) where the control law progressively reduced the speed according to the decrease of the value $P_{T}-P$ (Eq. (2)). Due to friction effects, the trajectories for reaching the different positions were not perfectly superimposed (see the box detail in Fig. 12). The maxi-mum repeatability of the time shift for reaching the same position, across 10 repetitions, was on average less than $10 \mathrm{~ms}$.

\section{Discussion}

\subsection{Clinical scenario of application}

Joining innovative robotics and mini-invasive surgical techniques appears to be a promising developing paradigm for treatment platforms in abdominal surgery over the next years. In our vision, NOTE surgery and dedicated miniature robotics devices would disclose the chance of developing new surgical techniques with the challenging aspect of relocating at the end the entire operating room within the abdominal cavity. Nonetheless, several steps are still necessary both in terms of techniques and technologies before this can become a reality. The work described in this paper is moving along this course focusing on technological issues of the endoscopic vision.

Traditional rigid endoscopes use expensive and damageable rod lenses to transport the image through a long tube to a backmounted camera. This increase the weight and make handling more difficult. Present developments in endoscopy aim for placing the optical imaging system together with the digital image sensor in the tip of the endoscope. A major drawback of these chip-onthe-tip endoscopes are their fixed lenses. Neither focusing nor optical zooming is possible. Frequent moving of the endoscope may result in missing anatomical features and consuming operation time.

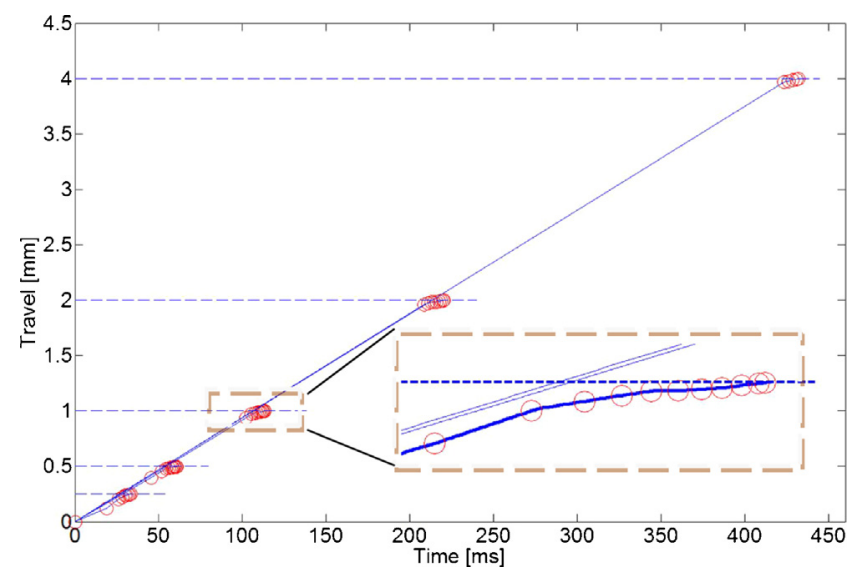

Fig. 12. Travel of 5 different distances, namely $0.25,0.5,1,2$ and $4 \mathrm{~mm}$. Red circles represent the point in the control loop when approaching the desired position. Detail of the motion steps when approaching $1 \mathrm{~mm}$. (For interpretation of the references to color in this figure legend, the reader is referred to the web version of this article.) 


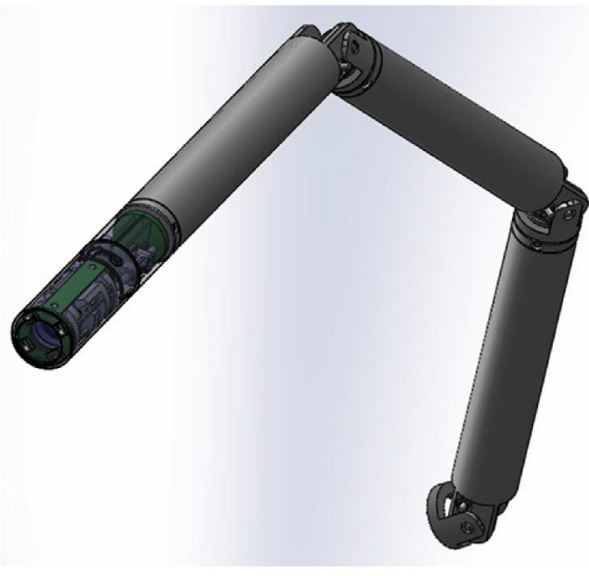

(a)

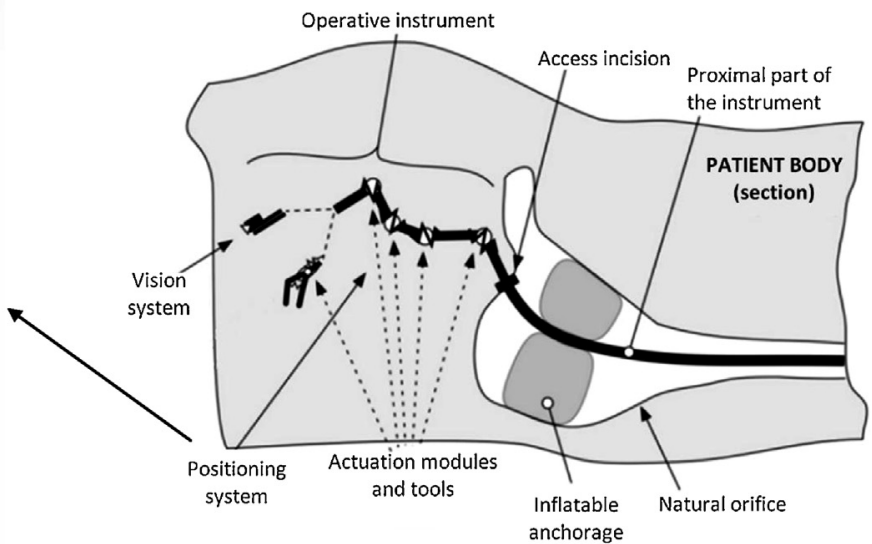

(b)

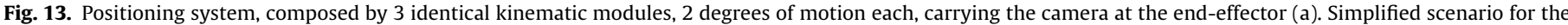

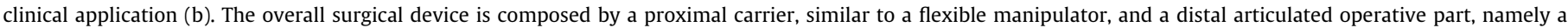
positioning system used to position and orient the vision system or alternatively the surgical tools.

We detailed the optical design and the lens displacement control of an innovative miniature vision system (patent pending) aiming at overcoming some limitations of the conventional endoscopic technologies. The designed system instead provides the chance of positioning the tip of the camera at variable distance from the target with a number of advantages. Increasing the distance to target reduces the interference with the operating tools and for instance can preserve the camera tip from getting dirty because of the fluid residual of cauterization. In addition, a greater FOV is obtained at the highest distance maintaining the image at focus. In comparison to long rigid endoscope $(20 \mathrm{~cm}$ long) the proposed system can be embedded into a $2 \mathrm{~cm}$ long cylindrical bulk.

A potential scenario for the application of the proposed apparatus was depicted in Fig. 13. The overall surgical instrument is composed by a proximal carrier, similar to a flexible manipulator, and a distal articulated operative part, represented as a chain of actuated kinematic modules to position and orient a surgical tool. This feature could contribute to overcome the limitation of the flexible endoscope used in NOTE surgery where the stiffness prevents easy maneuvering of the tip. The camera or any instrument, in principle, can be hooked to the end-effector of the distal operative part providing maximum flexibility of use and minimizing the needed surgical equipment.

\subsection{Optical design}

The development of the zoom system to be integrated within surgical tools decreasingly in size still remain an issue for at least four main reasons: (a) lens design cannot be simply obtained by scaling from development at macro-scale; (b) tolerances of the lens manufacturing at sub-millimeter size are difficult to warranty; (c) adopting mechanical compensation paradigm, the accuracy of the lens positioning is demanding and suitable actuation technologies cannot be readily available or are difficult to setup; (d) the fabrication of miniaturized lenses may be expensive on a short scale. In this paper, we addressed the first three issues by considering an ad-hoc lens design, which featured mechanical compensation, by analyzing the effects of tolerances on the optical performances, and by experimenting an innovative positioning technology, based on squiggle piezo-electric motors. We consider that the proposed work represents a fundamental methodological and technological step that can positively impact on the issue (d) in the former list reducing manufacturing costs and developing time.
The results of the optical design were basically consistent with constraints and specifications (Table 1 ). We obtained a 14 lens system, separated in four sets, with an overall length $L$ of $15.55 \mathrm{~mm}$ (technical specification $L<20 \mathrm{~mm}$ ) which was shown to be able to provide good optical performances in the distance range traditional of the surgical endoscopy setup. All the lens diameters were included in the specification range ( $2 \mathrm{~mm}<\varnothing<7 \mathrm{~mm}$ ). The attained maximum FOV, $56^{\circ}$ in the wide-angle zoom configuration, was in agreement with the requirements $\left(50^{\circ} \div 60^{\circ}\right)$. The lens set II, the variator, allowed obtaining a zoom factor of almost $2.8 \times$, whereas the lens set III, the compensator, allowed to attain almost con-stant image height and acceptable optical performances across the zoom range. As shown, in the worst condition a maximum astig-matism, spherical aberration and image distortion of $25 \mu \mathrm{m}, 35 \mu \mathrm{m}$ and $12 \%$, respectively, were predicted. The perceived sharpness was not lower than $65 \mathrm{lp} / \mathrm{mm}$. All design features and the optical per-formance were also in accordance with similar studies in literature [24]. The maximum lens travel distance was $3.30 \mathrm{~mm}$, for the zoom set, that was within the traveling range of the tested piezo-actuator. The tests on the tolerances reported in this paper were not com-prehensive of all the physical parameters but limited to the main quantities as lens curvature, relative axial distance, lens decentering and lens tilt. The analysis showed that the deviations to the nominal design conditions can dramatically decrease the optical performances. Interestingly, we found that greater attention should be paid to the tolerance of the compensation lens set than that one of the zoom lens set. Under the same tolerance conditions, a relative increase of more than $150 \%$ of the transverse aberration (see Tables 5 and 6 ) was predicted. In particular, the lens curva-ture tolerance was shown the most critical feature especially for the compensation lens set. Manufacturing tolerances for radius of curvature are typically $\pm 0.5 \%$, but can be as low as $\pm 0.1 \%$ in preci-sion applications. Recent lens micro-fabrication technologies have demonstrated the ability to cope with tolerances as low as $\pm 0.01 \%$ for extremely high quality needs [25]. Results showed that keeping the tolerance of the radius of curvature under $\pm 0.1 \%$ allows to approximate the nominal optical performances (Table 5). The manufacturing of the mechanical supports and opto-mechanics mounting is a critical issue as well. We attained that $\pm 1^{\circ}$ and $\pm 50 \mu$ $\mathrm{m}$ of lens tilt and decenter in the compensation set, respec-tively, deteriorate the transverse aberration of about $80 \%$ and $20 \%$ (Table 5 ). Therefore, precise techniques for the micro-fabrication of the mechanical supports are mandatory to assure high reliability in 
the relative coupling among the mobile parts and to minimize the friction.

\subsection{Lens positioning control}

A weakness of moveable lenses is that individual elements of these optical systems have to move very precisely along calculated trajectories. The control of lens motion was experimentally investigated using the miniature squiggle motor and magnetic position encoders in a closed-loop control setup. The technical features of the motor (linear positioning resolution: $0.5 \mu \mathrm{m}$; traveling path: $6 \mathrm{~mm}$ ) were verified showing that it can travel millimeter path $(3.5 \mathrm{~mm})$, with micrometric accuracy $(5 \mu \mathrm{m})$ as required for positioning lens sets, in reasonable time. In comparison to other piezo-actuators $[20,21]$, the selected motor can be readily integrated into low power system as it can be actuated using $3.3 \mathrm{~V}$ voltage thus avoiding dedicated power adaptation.

\section{Conclusions}

The performance evaluation of the predicted image quality and the positioning feature of the squiggle motor can meet the requirements of a miniaturized optical systems for laparoscopic surgery. We acknowledge that, although optical design can be simulated very effectively, there still exist significant differences between a fabricated system and the simulation due to manufacturing and assembly tolerances. However, the fabrication of such a complex optical system can take advantage from design studies similar to that one presented in the present paper. Overall this paper can be considered a significant contribute toward future developments of integrated miniaturized camera systems for biomedical applications.

\section{Funding}

This work was supported in part by the Italian Ministry of Research and University under Grant PRIN “2008KJWNNW_003”.

\section{Ethical approval}

Not required.

\section{Competing interests}

None declared.

\section{Appendix}

\section{Mechanical compensation paradigm}

Mechanical compensation in its simplest arrangement consists of two mobile optical sets. The first one called "variator" is responsible for zooming but introduces defocus and optical aberration. In order to recover the focus loss, a second mobile lens set, called "compensator", is used. Actually, traditional zoom optics include either one or two additional fixed optical sets, to reduce aberrations and increase overall optical power. For miniaturization purposes, these two aspects are significant. Considering a four lens set optical system (see Fig. 1), L1 is the front fixed set, L2 is the mobile zoom set, L3 is the mobile compensation set and L4 is the rear fixed set. Each set is represented by an equivalent focal length and a magnifi-cation factor, namely $f$ and $m$, respectively. The set distances are $d_{12}, d_{23}, d_{34}$. When the optical system moves from short to long focus set L2 travels to the right while set L3 travels to the left, shortening $d_{23}$ and broadening both $d_{12}$ and $d_{34}$. In order to assure optimal focusing at different magnification factors and avoid lens impact, distances should be properly chosen.

In this study, the chosen optical format was positivenegative-positive-positive, namely negative for zooming and positive for compensation. Then, the magnification $m_{3 L}$ of compensation set at long focus can be computed as:

$m_{3 L}=\frac{f_{3}}{f_{2}\left(1-m_{2 L}\right)-d_{23 L}+f_{3}}$

Assuming $f_{2}=-1$ (reference value) and $\left|m_{3 L}\right|<1$, a superior $\operatorname{limit}$ for $f_{3}$ is:

$f_{3} \leq 0.5\left|\left(f_{2}\left(1-m_{2 L}\right)-d_{23 L}\right)\right|$

The object distance $l_{2 L}$ and image distance $l_{2 L}^{\prime}$ of the zoom set the object distance $l_{3 L}$ and image distance $l_{3 L}^{\prime}$ of the compensation set can be expressed as:

$$
l_{2 L}=f_{2}\left(\frac{1}{m_{2 L}}-1\right) \quad l_{2 L}^{\prime}=f_{2}\left(1-m_{2 L}\right)
$$

$l_{3 L}=l_{2 L}-d_{23 L} \quad l_{3 L}^{\prime}=\frac{l_{3 L} f_{3}}{l_{3 L}+f_{3}}$

with $m_{3 L}=l_{3 L}^{\prime} / l_{3 L}$.

Considering a position change of the zoom and compensation sets and assuming that $D$ is constant then the object and image distances are changing as:

$$
\begin{aligned}
& l_{2}=f_{2}\left(\frac{1}{m_{2}}-1\right) \quad l_{2}^{\prime}=m_{2} l_{2} \\
& l_{3}=0.5\left(-L_{3}+\sqrt{L_{3}\left(L_{3}-4 f_{3}\right)}\right) \quad l_{3}^{\prime}=L_{3}+l_{3}
\end{aligned}
$$

Given that $f_{1}=d_{12 S}+l_{2 S}$ and $f_{2}=d_{34 S}-l_{3 S}$, the three moving distances are computed as:

$d_{12}=f_{1}-l_{2} \quad d_{23}=l_{2}^{\prime}-l_{3} \quad d_{34}=l_{3}^{\prime}-l_{3 S}^{\prime}+d_{34 S}$

In order to avoid lens set impact, we initially defined $d_{23 L}=0.05 \mathrm{~mm}$ (minimal distance between zoom set and compensation set in the long focus condition), $d_{12 S}=0.05 \mathrm{~mm}$ (minimal distance between the zoom set and the first set in the short focus condition), $d_{34 S}=0.05 \mathrm{~mm}$ (minimal distance between the compensation set and the fourth set in the short focus condition). Setting a reasonable value for $f_{2}$ and $m_{2 L}$ leaded to obtain an initial set of focal lengths, magnification factors and lens set distances. From them we attempted different lens set typologies according to the chosen optical format.

\section{References}

[1] Bessler M, Stevens P, Milone L, Parikh M, Fowler D. Transvaginal laparoscopically assisted endoscopic cholecystectomy: a hybrid approach to natural orifice surgery. Gastrointest Endosc 2007;66:243-1245.

[2] Marescaux J, Dallemagne B, Perretta S, Wattiez A, Mutter D, Coumaros D. Surgery without scars. Arch Surg 2007;142:823-6.

[3] Forgione A, Maggioni D, Sansonna F, Ferrari C, Di Lernia S, Citterio D, et al. Transvaginal endoscopic cholecystectomy in human beings: preliminary results. J Laparoendosc Adv Surg Tech A 2008;18:345-435.

[4] Menciassi A, Quirini M, Dario P. Microrobotics for future gastrointestina endoscopy. Minim Invasive Ther Allied Technol 2007;6(2):91-100.

[5] Rentschler M, Dumpert J, Platt S, Farritor S, Oleynikov D. Mobile in vivo robots provide sole visual feedback for abdominal exploration and cholecystectomy. Surg Endosc 2006;20(1):135-8.

[6] Zoppi M, Molfino R, Cerveri P. Modular micro robotic instruments for transluminal endoscopic robotic surgery: new perspectives. In: Proceedings of 2010 IEEE/ASME international conference on mechatronic and embedded systems and applications (MESA). 2010. p. 440-5.

[7] Lehman AC, Dumpert J, Wood NA, Visty AQ, Farritor SM, Oleynikov D. In vivo robotics for natural orifice transgastric peritoneoscopy. Stud Health Technol Inform 2008; 132:236-41.

[8] Shaikh SN, Thompson CC. Natural orifice translumenal surgery: flexible platform review. World J Gastrointest Surg 2010;2:210-6. 
[9] Zazzarini CC, Pansini A, Zaltieri R, Forgione A, Pugliese R, Cerveri P. Design of a robotic endoscope for mini invasive surgery. In: Proceedings of the ASME 2011 international design engineering technical conferences \& computers and information in engineering conference. 2011. p. 771-7.

[10] Hu T, Allen PK, Hogle NJ, Fowler DL. Insertable surgical imaging device with pan, tilt, zoom, and lighting. Int J Robot Res 2009;28(10):1373-86.

[11] Fowler DL, Hu T, Nadkarni T, Allen PK, Hogle NJ. Initial trial of a stereoscopic, insertable, remotely controlled camera for minimal access surgery. Surg Endosc 2010;24:9-15.

[12] Wang RC, Deen MJ, Armstrong D, Fang Q. Development of a catadioptric endoscope objective with forward and side views. J Biomed Opt 2011;16(6):066015.

[13] Nakamura T, Terano A. Capsule endoscopy: past, present, and future. J Gastroenterol 2008;43(2):93-9.

[14] Yamaji K. Design of zoom lenses. In: Wolf E, editor. Progress in optics. Amsterdam: North Holland; 1967. p. 105-70.

[15] Demenikov M, Findlay E, Harvey AR. Miniaturization of zoom lenses with a single moving element. Opt Express 2009;17(8):6118-27.

[16] Demenikov M, Findlay E, Harvey AR. Experimental demonstration of hybrid imaging for miniaturization of an optical zoom lens with a single moving element. Opt Lett 2011;36:969-71.

[17] Bellouard Y. Shape memory alloys for microsystems: a review from a material research perspective. Mater Sci Eng A 2008;481(482):582-9.
[18] Clausi D, Gradin H, Braun S, Peirs J, Stemme G, Reynaerts D, et al. Design and wafer-level fabrication of SMA wire microactuators on silicon. J Microelectromech Syst 2010;9(4):982-91.

[19] Clausi D, Gradin H, Braun S, Peirs J, Stemme G, Reynaerts D, et al. Robust actuation of silicon MEMS using SMA wires integrated at wafer-level by nickel electroplating. Sens Actuators A 2013;189:108-16.

[20] Karpelson M, Wei G-Y, Wood Robert J. Driving high voltage piezoelectric actuators in microrobotic applications. Sens Actuators A 2012;176:78-89.

[21] Guo M, Dong S, Ren B, Luo H. A piezoelectric single-crystal ultrasonic microactuator for driving optics. IEEE Trans Ultrason Ferroelectr Freq Control 2011;58(12):2737-42.

[22] Patronik N, Ota T, Zenati MA, Riviere C. A miniature mobile robot for navigation and positioning on the beating heart. IEEE Trans Robot 2009;25(5):109124.

[23] Park JT, Lee JG, Kim SY, Kim GH, Hu KS, Cha JY, et al. A piezoelectric motorbased microactuator-generated distractor for continuous jaw bone distraction. J Craniofac Surg 2011;22(4):1486-8.

[24] Kuiper S, Hendriks BHW. Variable-focus lens for miniature cameras. Appl Phys Lett 2004;85(7):1128-30.

[25] Wang Q, Cheng D, Wang Y, Hua H, Jin G. Design, tolerance, and fabrication of an optical see-through head-mounted display with free-form surface elements. Appl Opt 2013;52(7):C88-99. 